\title{
Evaluating Library Discovery Tools through a Music Lens
}

\section{Rebecca Belford}

This paper outlines how to use specialized cataloging to evaluate discovery tools for library collections. An awareness of the capability offered by bibliographic records and data for specialized materials enables libraries to conduct their own evaluation of a discovery tool's functionality for complex materials. Bibliographic records for materials such as music are excellent for putting a discovery tool through rigorous testing. This paper may be used to facilitate evaluation of discovery tools by those without music cataloging expertise, and it provides a ready supply of examples to quickly identify complex search strategies.

Throughout this paper, the term discovery tool refers to products that meet the criteria of web-scale discovery. Concisely defined by Hoeppner, these are the central index, a preharvested central index of content, and the discovery layer, the user interface to the central index. ${ }^{1}$ The meaning of discovery tool varies throughout the library literature and has, as documented by Caplan, changed over time; ${ }^{2}$ vendors may refer instead to a service or solution. Little compares these to the online public access catalog (OPAC) and refers to these as "softer, gentler OPACs." ${ }^{3}$ Discovery tools currently available to libraries include VuFind (open source, developed and maintained at Villanova University), Blacklight (open source), BiblioCore (BiblioCommons), Summon (Serials Solutions), Primo (Ex Libris), EBSCO Discovery Service (EBSCO), and WorldCat Local (OCLC). ${ }^{4}$

Central index coverage is variable and customizable. It includes a library's resources and may include local digital collections or institutional repositories, subscription databases, and external open access collections. It is the MARC records within a discovery tool's data that are the focus of this paper. Characteristics of the discovery interface that are consistent across all tools are a single search of the central index, a simple keyword search box, relevancy ranking, facet or refinement tools, and recommendations or spell check; additional features vary between products or libraries. ${ }^{5}$ Topics covered in this paper may address the interface (display), central index (indexing), and areas where they intersect. Browse searching and visible use of authority data are standard features of many OPACs that are not emphasized or available in every discovery tool, ${ }^{6}$ and these features are not discussed in this paper. However, the questions and search strategies provided may be used to evaluate OPACs to the extent that features overlap.

Rebecca Belford (rbelford@buffalo.edu) is Music Cataloger/Reference Librarian, State University of New York at Buffalo.

Submitted November 30, 2012; revisions submitted July 12, 2013; tentatively accepted with minor revisions August 2, 2013; accepted for publication September 13, 2013. 
When individual libraries or reviewers evaluate a discovery tool, they typically employ simple searches that replicate those of a generalized set of users. Vendors often demonstrate their own products with preselected searches guaranteed to produce attractive results. The practice of using simple searches will evaluate how the basic needs of a majority of users are met, and it will demonstrate how a discovery tool handles simple bibliographic records. This practice does not demonstrate how a discovery tool handles complex topic searches or known-item searches that may be required for specialized materials. The needs of users of specialized materials may not emerge unless a tool is subjected to stringent testing. A tool that provides the indexing, search, and display features that allows users to discover specialized materials will meet the basic needs of the specialist user while easily meeting and surpassing the needs of the general user.

\section{Evaluation through a Music Lens}

In this paper, music is used as an exemplar of specialized materials that require the more rigorous evaluation of a discovery tool. The description of how music data can be used for rigorous evaluation of a discovery tool will serve as a resource for catalogers (and others who are comfortable with MARC and cataloging terminology) who may not regularly catalog or search for music materials but are involved in selecting a discovery tool that includes records for music resources.

Bibliographic records for music materials are often complex. The underlying challenges of music description include the existence of multiple manifestations and expressions of a single work and the related need to collocate works and expressions. ${ }^{7}$ Multiple works, expressions, creators, and contributors may be represented in a single resource, making relationships difficult to reflect clearly in a single record. Additional challenges include describing form, genre, and medium, accounting for a multilingual publishing environment, and providing identification of various formats of materials. Users of music materials may seek materials for a specific instrument or by a particular performer, or they may require a precise "known-item" query.

The Music Library Association (MLA) has published a document detailing system-neutral search, indexing, and display requirements for the discovery of music resources. ${ }^{8}$ Organized loosely around the Functional Requirements for Bibliographic Records (FRBR), "Music Discovery Requirements" includes discussions of individual attributes of musical works, expressions, and manifestations, accompanied by an explanation of the importance of being able to effectively search for and view this information for the discovery needs of music users. The intended readership is broad; it targets "those creating or guiding the development of discovery interfaces that will include music materials." ${ }^{\prime 9}$ The document's indexing and display recommendations can be used to create or adapt a discovery tool; however, it does not provide recommendations or examples of how one might evaluate an existing product.

This paper provides readers with a practical method to evaluate individual discovery tools. Theoretical issues related to the description of music materials or reasons why certain features of a discovery tool are critical for music are not addressed in this paper. For additional explanations of the relationships between user needs and discovery tools, readers are urged to consult the corresponding sections of the MLA document.

\section{MARC Records: Evaluating a System from the Inside Out}

MARC records will remain in libraries' databases for the near future, even as the Library of Congress's Bibliographic Framework Transition Initiative (BIBFRAME) is under development. ${ }^{10}$ Discovery tools can include records created with multiple metadata encoding schemes, including Dublin Core (DC) records from digital collections or Encoded Archival Description (EAD) finding aids. Even though MARC records are a subset of records searched by a discovery tool, their examination is critical. A discovery tool's degree of smaller-scale functionality - display, indexing, simple hyperlinking-for MARC records can be indicative of its ability to realize larger-scale functionality such as features that maximize FRBR capabilities, utilize authority data, or incorporate nonlibrary data. A product's treatment of its fullest and most complex MARC records will illustrate its capacity to harness fully the powerful capabilities of bibliographic data. An investigation based on MARC data will help answer this question: Is a discovery tool realizing its full potential?

Fully evaluating how well discovery tools utilize bibliographic data to power searches and represent resources requires familiarity with the MARC structure and records that generate public displays. By default or design, not all areas of bibliographic records display in every system, search terms might not display in results, and contents of fields and subfields may be suppressed or rearranged. For these reasons, records retrieved in public interfaces must be compared against MARC records. Comparison methods depend on the discovery tool and its configuration. In some public interfaces, a labeled MARC record can be viewed by selecting a "staff" or "librarian" link. Other interfaces may not offer a publicly viewable MARC record, and it may be necessary to view the same record in the staff module of the integrated library system (ILS), or, in the case of OCLC's public WorldCat interfaces, in Connexion or 
another OCLC subscription product. The comparison of the public display against its corresponding MARC record allows for a thorough investigation using bibliographic data and ensures that it is the discovery tool that is being evaluated, not the bibliographic data itself. Direct exploration of MARC records is ideally accompanied by access to system information including indexing tables and display rules. The method described in this paper can compensate if system information is unavailable, or it can be part of a multifaceted assessment.

\section{Checklists and Evaluation Cycles}

A flexible checklist can be used to evaluate and customize a discovery tool throughout its life cycle. Depending on its users and vendors, each library will need to develop a checklist that best meets its needs. Before implementation, a checklist can be used during the request for proposals and selection processes. A checklist allows a library to compare vendors' versions of discovery tools and the most current versions of multiple tools as implemented at various institutions. Following selection, a checklist can be used to request enhancements and customizations from vendors or in-house programmers; libraries that are beta testers or development partners can also use a checklist to shape a new system. A library can use a checklist for periodic reevaluation of a system to see if it still works as desired. When enhancement options are exhausted or the system no longer meets a library's needs, a checklist can be used to begin a new selection process.

A good checklist is flexible and may be customized locally. Checklist items that involve bibliographic records will need revision as cataloging practices change and evolve. Discovery tool features can change frequently based on emerging needs, and products may enter or leave the market. Users' and libraries' expectations of functionality and design change over time. Libraries should adjust discovery tool requirements and evaluation measures based on their own unique collections and user needs.

\section{Potential Effect of RDA and Changing Standards}

The ongoing relationship between the implementation of Resource Description and Access (RDA) and discovery tools is difficult to predict. MARC format has been changed to accommodate RDA. In most cases, new fields and subfields have been defined; in a few cases, fields or subfields will become obsolete. As RDA records are added to a database, modifications will be required to add or adjust display and indexing for records to continue to reflect the same type of information (i.e., a newly defined MARC field replaces a newly obsolete field for recording identical content). The continued use of MARC with RDA means that RDA's emphasis on relationships and linkages may be difficult to express in current discovery tools.

Changed rules for constructing access points mean that without retrospective conversion of Anglo-American Cataloguing Rules, 2nd edition (AACR2) access points in bibliographic records to RDA, different access points for the same entity may coexist in the database, linked only by a "see" reference in the RDA authority record. This illustrates the importance of authority records, currently underutilized by discovery tools. In an RDA environment, the use of authorized access points are essential to reflecting FRBR relationships, and bibliographic records no longer have restrictions on added access points as under AACR2 (e.g., no restrictions on the number of collaborators represented in access points). Use of controlled access points for works and expressions and the creation of authority records are long-established practices in music cataloging, meaning that existing records for music resources provide a rich source of test material.

In addition to the implementation of RDA, records for music materials will be affected by the implementation of the Library of Congress (LC) Genre/Form Terms for Library and Archival Materials (LCGFT) and the Library of Congress Medium of Performance Thesaurus (LCMPT) for music. These thesauri will replace the use of LC Subject Headings (LCSH) to provide access to form, genre, and medium for musical works. ${ }^{11}$ The LCMPT required the definition of new MARC fields, and indexing and display rules in end-user and back-end tools may need to be changed to accommodate the MARC format change. As with all MARC changes, libraries may make individual decisions regarding implementation and timetables in their ILS or discovery tool; as with previous headings changes and LCGFT implementations, libraries may retain older headings in existing records.

\section{Literature Review}

The literature relevant to the selection and evaluation of discovery tools includes usability studies, case studies, reviews, and evaluative checklists. Beginning with the earliest days of ILSs and OPACs, librarians have used their familiarity with data capabilities and users' needs to develop checklists for selection, evaluation, and improvement of library discovery interfaces. In describing evaluation of OPACs in the 1980s, O'Rourke advocates for the checklist approach. One advantage is that "by systematically examining catalog characteristics, a library comes to define its own catalog requirements more accurately and adequately." ${ }^{\prime 2}$ Luong and Liew share 
the process and checklist used to evaluate traditional OPACs in academic libraries in New Zealand. They offer tables with detailed search and display criteria that staff used to evaluate multiple products. Many of these criteria are of high importance to the discovery of items with complicated bibliographic descriptions: the use of cross-references, authority control, and logical display of fields within bibliographic records. ${ }^{13}$

Today, with the emergence and development of webscale discovery tools, checklists continue to be a powerful evaluative tool. Vaughan describes the process of involving library staff in selecting a web-scale discovery service at the University of Nevada Las Vegas Libraries. The process included a "staff survey" related to multiple aspects of webscale services; the questions on the end user interface are appropriate for discovery tools of any kind. ${ }^{14}$ Ramsay and Chamberlain offer a broad list of selection considerations emphasizing open-source tools in their "Software Selection Methodology for Library Discovery Layer Systems." ${ }^{\text {"15 }}$

Specific products have been studied and compared in the course of selection and implementation at individual libraries or consortia, and as stand-alone discovery tools not tied to any particular library. Yang and Wagner compared seventeen discovery tools against a checklist of features that characterize next-generation catalogs and discovery tools. Although features can change rapidly as products evolve, features on their checklist remain standard in discovery tools. ${ }^{16}$ The literature includes numerous case studies that describe the implementation of discovery tools at individual libraries, many of which include details of the selection process as well as discussions of the tools considered. Individual discovery tools reviewed or evaluated in the context of music discovery include Summon, WorldCat Local, and Primo. $^{17}$

Usability testing has been conducted in a number of libraries (primarily academic). Fagan's literature review of usability studies describes the study and results at each university library represented, followed by recommendations for the method for future studies. Fagan emphasizes that study results are affected by the scope and size of individual collections. ${ }^{18}$ Studies focusing on music users have also been conducted. Music faculty and graduate students were the subjects of a usability study of the next-generation catalog AquaBrowser (Serials Solutions) in use at the University of Chicago, ${ }^{19}$ and usability testing of OCLC's new user experience for FirstSearch with music graduate students is planned at the University of Washington. ${ }^{20}$ Transaction logs provide another source of evaluation, using large-scale data to analyze searches and features used. Search-log studies include a comparison of use of the classic OPAC and the discovery tool Encore (Innovative Interfaces) at New York Law School reported by Ballard, and Meadow and Meadow's study of the transaction logs from Summon at Montana State University. ${ }^{21}$

\section{Method}

The strategy here offers concrete methods for analyzing a discovery tool's use of the data contained in existing bibliographic records for music material. Selected specific elements of MARC records that are particularly important to utilize for users to discover and select music materials are discussed. Search strategies are included, often with suggested search strings. Searches are intended to work in a general keyword index (the default simple-search-box option standard in discovery tools), with limits (pre-search), facets (post-search), or other indexes indicated as appropriate. If searches are replicated in a web-scale discovery tool that includes data from multiple sources, limiting searches or results to bibliographic ("catalog" or "library") records will focus examination on MARC records. Search strings are enclosed in curly brackets \{like this\} to avoid confusion as to whether quotation marks are part of the search. Searches containing multiple terms are assumed to be joined as a Boolean AND search. Comparison of the public and MARC records may be necessary to determine why results were retrieved in a search, what controlled title access points are present, and how titles are recorded and encoded.

Appendix A can be used or adapted as a checklist. It contains questions related to record elements and system functionality, including both general features and musicspecific ones. Appendix B contains specific recommended searches, sample access points in MARC format, or titles with OCLC numbers and relevant fields from bibliographic records. Appendix B is meant for in-depth analysis of a system's handling of music materials. Examples are exclusively music-based, though many elements of headings or records are shared by other materials. They may also be used without interpretation as MARC examples for programmers; the only additional step necessary would be to confirm that authorized access points match the LC authority file. ${ }^{22}$

\section{Beginning the Evaluation}

\section{General Features}

A thorough direct exploration of discovery tools begins with a consideration of features related to the system as a whole. This will place scrutiny of individual bibliographic records in context. Features that merit consideration include search options, facets, treatment of physical formats, the linking of controlled fields, available indexes, and display of the content of records and encoded information. Within these categories, certain features are of particular importance for music. Ideal functionality will also provide a direct benefit for nonmusical materials.

End users begin searching and refining results through 
basic and advanced search options, post-search facets, and lists of results. Familiarity with these features enriches understanding of the end-user experience. After examining search options, a large set of search results will enable investigation of additional characteristics of the discovery tool. To retrieve a large set of results, a blank search (without search terms) is ideal. Broad searches such as $\{$ history\}, $\{$ Bach $\}$, or \{music\} are also effective. A spot-check of individual results will give a general picture of layout, labels, navigation, facets, item details, and any other features of interest.

\section{Specific Fields and Elements for Investigation}

Records for music materials can include format information, multiple languages, genre/form and subject challenges, and the representation of multiple creators, contributors, works, and expressions. Bibliographic information in the following areas is discussed:

- creator and contributor names

- titles of works and expressions

- analytic entries

- relationships

- subject subdivisions and facets

- genre/form

- medium of performance

- physical description

- format options

- languages

- notes (language/notation, performer/cast, production, recording, contents)

- edition information

- numbers (publisher, music, or plate)

- diacritics, special characters, and stopwords

Each area is addressed, including general search and evaluation strategies. Specific questions for use in a checklist and detailed searches and examples are provided in the appendixes.

\section{Authorized Access Points and Display of Creator and Contributor Names}

The need to accurately represent creators and contributors in a discovery tool is universal across subjects and formats. Challenges related to display and linking functionality are compounded in music records, where it is common for more than one creator or contributor to be associated with a resource. Records for scores may contain added access points for an editor of the music, who provides a substantial intellectual contribution, or for collaborators (such as librettists) for dramatic works. Records for music sound recordings present more complex challenges. For a single classical work, there will be an access point for the performer in addition to one for the musical work. In compilations, there may be multiple works by different composers, and different performers for each work. In all cases, the forms of names in access points do not necessarily match those in statements of responsibility or notes fields.

Post-search facets, lists of results that display following a search (henceforth referred to as "brief results"), displays of single records, and linking functionality on controlled access points are areas where a discovery tool's treatment of name access points requires evaluation. The display of names in brief results and individual records has proved problematic in discovery tools. Default settings and customizations vary widely with products and instances. Faceted tools may not automatically include all traced creators or contributors under an author facet.

To determine whether or how corporate names appear under an author facet, one may conduct an author index search for all or part of an authorized access point for a prolific or locally represented musical group. If there is no author index option, one may substitute a general keyword search for the name limited to a sound or video recording format or with a format-related search term such as "disc." In all-field keyword searches, a phrase search may be required in cases where individual elements of a name are likely to appear separately (\{“Chicago Symphony”\} to exclude results with "Chicago" in a location note and "symphony" somewhere else). Corporate names where a group is identified as a subordinate unit may be misrepresented in a facet. Only the parent body might display: "Bayerischer Rundfunk" versus "Bayerischer Rundfunk Orchester" (1102\# \$a Bayerischer Rundfunk. \$b Orchester).

A list of brief results for records with multiple name access points will indicate where information from name access points is visible and how it is labeled. A brief results list can be retrieved with a simple search for a musical form, performer, or composer. In individual records, one should check the following: where names or portions of names from access points appear, whether they appear in separate locations within a record, how they are labeled, how they are grouped, and whether authorized name access points replace the statement of responsibility in MARC field 245 $\$ c$. Searches with elements of the authorized form of a name used as keywords will test indexing as well as display. The display of names where authorized access points are entered in direct order (a form other than "last name, first name") should also be investigated.

All controlled access points serving as hyperlinks should function as intended. For names, this means referring to the correct individual or body. The challenge of precision can be most acute with common names (such as John Adams) or names based on given names (such as Hildegard of Bingen), where additional name access point elements beyond 
the information recorded in $\$$ a of name headings (dates, numeration, etc.) are needed to identify or link to the correct entity. Links should be tested both in facets and within records because some discovery tools generate different searches depending on what screen a user views.

\section{Titles of Works or Expressions}

The importance of consistent, controlled access points or identifiers for musical works cannot be overstated. They are essential for the indexing and collocation of musical works and expressions, even if end users do not use them as search terms or see them. Discovery tools vary widely in how these titles are displayed or utilized for linking or collocation. Some may completely suppress these fields by default, while others may provide partial or full display or linking.

The titles illustrated in this paper are the standard form used to consistently identify, differentiate, and collocate musical works and expressions. The term preferred title is used to reflect RDA terminology and is the successor to AACR2's uniform title. In MARC bibliographic records, these titles are those found in access points in fields 130, 240, 730, and title subfields (\$t with additions) in 700, 710, or 711. Functionality related to preferred titles includes indexing (title, keyword, or other), visibility in a record, fullness and accuracy of display, association with appropriate names, and any linking or collocation feature. Because treatment of preferred titles is so fundamental to determining the adequacy of a discovery tool for music, these titles are discussed in greater detail than are the remaining sections.

For preferred titles to function as unique identifiers for works and expressions, all elements within the title are important to ensure accurate identification and differentiation. For preferred title search strings to link precisely to a work or expression, every subfield, in order, is needed. If full titles are displayed to users, subfields should display in their proper order. Displayed titles for works that have creators (composers) integral to access points (titles in fields 240, 700,710 , and 711) must be associated with those names to be meaningful to users, and titles must be bound to those names to provide linking functionality based on works and expressions.

Music cataloging divides preferred titles into two categories: generic (a type of composition, such as "symphony") and distinctive (those that are not generic). A typical configuration of a distinctive title in a name-title access point is in the format "Mozart, Wolfgang Amadeus, $\$ \mathrm{~d}$ 1756-1791. \$t Zauberflöte." To execute searches for distinctively titled works where the authorized form of the title and a transcribed or translated title are likely to differ, one may begin with the authorized form or a transcribed form. Searching by the authorized form-e.g., \{Zauberflöte\}permits simultaneous evaluation of display and indexing, but requires knowledge of the authorized term. Searching by a transcribed or common English-language form-\{Magic Flute\}-requires careful scrutiny of the displayed record for the authorized form. Records for works with generic titles will be retrieved with keyword searches for the composer's name plus known elements of the preferred title (i.e., form, medium, or opus number). In some cases, they can be retrieved with searches for the composer's name plus the work's commonly known name.

Titles in authorized access points for musical works may include additional elements for differentiation to indicate expression-level characteristics or to indicate whole-part relationships. A single access point can contain elements and subfields representing all of these categories. Subfields following $\$ \mathrm{a}(240$, most commonly) or $\$ \mathrm{t}(7 \mathrm{xx})$ indicate medium $(\$ \mathrm{~m})$, work or serial number $(\$ \mathrm{n})$, and key $(\$ \mathrm{r})$, with a typical configuration in the form "Beethoven, Ludwig van, $\$ \mathrm{~d}$ 1770-1827. \$t Sonatas, $\$ \mathrm{~m}$ violin, piano, $\$ \mathrm{n}$ no. 5, op. 24 , \$r F major." Preferred titles can also indicate whole-part relationships. Parts may be numbered $(\$ \mathrm{n})$ or named $(\$ \mathrm{p})$, and these subfields can be repeated to indicate named or numbered parts of parts. Additional complex configurations are possible. Expression-level characteristics that may be appended to preferred titles of works include language $(\$ 1)$, version $(\$ \mathrm{~s})$, and arrangement $(\$ \mathrm{o})$. The use of "selections" $(\$ \mathrm{k})$ is commonly used to indicate excerpts of a single work or selected works in a composer's body of works.

When examining linking functionality of title access points with multiple subfields, it is necessary to explore what degree of linking a discovery tool offers. Some discovery tools may not provide links on title access points. Some generate links from the first subfield only ( $\$$ a in 130/240/730 or $\$$ t in $700 / 710 / 711$ ), while others generate bound links on the entire title-access-point string to the exact expression represented. Associated names may not be linked to titles by default, or only the names may be links.

The display, linking, and indexing of access points for preferred titles constructed without names should also be explored. Records for most motion picture soundtracks include an added access point for the preferred title for the film. A keyword search limited or narrowed to sound recordings for \{motion picture music [film title]\} should lead to records with the film title as an added access point in the MARC 730 field. Access points for musical works without names are less common.

\section{Analytic Entries}

Bibliographic records for sound recordings containing musical works (Western art music) by multiple composers or works of different types by a single composer typically contain analytic access points, which are access points for a work or expression contained completely in the resource 
described. Records with analytic authorized title access points are relatively easy to find, e.g., compact discs with works by more than one composer. Searches that are most likely to locate records with analytic entries include those for multiple composers' names, one composer with two named or nicknamed works (Mozart's "Haffner" and "Jupiter" symphonies), or titles frequently issued together (the operas Cavalleria and Pagliacci). A "sound recording" limit or facet creates a more manageable set of results, particularly with general keyword index searches.

\section{Relationships}

The role of a person or corporate body is important for the identification and selection of music materials. This is particularly important for sound recordings, where composers and performers may be of equal importance to users, or where it needs to be clear that a single person is both the composer and performer. Roles can be indicated in notes, or can follow name access points in MARC relator codes (\$4), optional in AACR2 records, or in relationship designators (\$e) defined in RDA. Testing the use of codes is still necessary if codes in $\$ 4$ remain unconverted to terms in $\$$ e. A decision about display of codes and terms may depend on how many records in a library's database contain the codes and whether new records include codes or terms. If codes or terms have not been applied consistently, display and indexing in discovery tools will not accurately represent relationships potentially described in those subfields for all records.

The representation of work-to-work relationships as defined in FRBR and RDA are common with dramatic musical works (operas based on existing plays) and vocal works (preexisting texts or poems later set to music). Preferred titles of related works are typically included in bibliographic records for the newer work. Access points in AACR2 records cannot indicate the precise nature of the relationship, meaning the information is provided in a cataloger-supplied note. In RDA records, work-to-work relationships are indicated by preceding an access point with relationship information in $\$ \mathrm{i}$ in a MARC 7xx field. The access point "7001\# $\$ \mathrm{i}$ Based on (work): \$a Shakespeare, William, \$d 1564-1616. \$t Romeo and Juliet" would be present in records for the various inspired works by Bellini, Berlioz, Gounod, Lavrovskiĭ, Prokofiev, Rota, Tchaikovsky, and Zeffirelli. To confirm that contents of $\$ \mathrm{i}$ are not in author or title indexes, one would search for the relationship term in each index, avoiding terms that are identical to words used in name authorized access points, particularly in $\$ \mathrm{c}$ (e.g., instrumentalist). If $\$ \mathrm{i}$ in MARC 7xx name or title fields is in these indexes, there will be results from title or author index keyword searches for \{based work\} or \{"based on work"\}. Similar searches with \{contains work\} or \{contains expression\} will address the use of $\$ \mathrm{i}$ in analytic titles in the MARC 7xx field.

\section{Subject Subdivisions and Facets}

Current LCSH for notated or recorded music frequently employ form ( $\$ \mathrm{v}$ ) subdivisions ("Scores and parts") or chronological (\$y) subdivisions (“1991-2000”). Faceted discovery tools offer different default and customized arrangement and indexing of subject headings in facets. Topical subdivisions in a single subject heading may be separated within a subject facet, distributed among different facets, or suppressed from view. Topical headings and subdivisions typically appear under "subject" or "topic." Form, chronological, and geographic subdivisions may appear in other facets such as "genre" or "era," based on subfield coding, or they may be completely omitted from facets. Form subdivisions for notated music in LCSH and representation of notated music coded in the MARC LDR, 00x, or 336 fields can closely correlate ("Scores") or be more precise ("Scores and parts"). ${ }^{23}$ Because of this overlap, "Scores" may appear twice in facets, such as format and subject or format and genre. When investigating facets, apparent duplicates may be the result of separate coding or fields generating each term.

Preferred titles are rarely, if ever, included by default in subject facets. To investigate, one would search by the authorized form of title for works or groups of works by a composer likely to be the topic of book-length analysis, such as Wagner's Ring opera cycle, or by keywords that include a composer's name and subdivision terms such as analysis appreciation\} that imply topical treatment of a work. Distinctive titles are preferable for these searches. In some cases the initial element of a preferred title ("Requiems, D minor") may be identical to the topical term ("Requiems"), making it difficult to differentiate subject keyword indexing of topical subjects from preferred titles as subjects. Linking functionality of subjects that are work titles requires similar evaluation to that for title access points coded as titles, e.g., titles in a MARC 700 field.

\section{Genre/Form}

The dedicated genre/form MARC field (655) is currently used in records for music resources such as music-related films or radio programs because LCGFT has already been implemented for moving images and radio. Most genre/ form information in records for music scores and recordings is represented in subject headings in the MARC 650 field, Subject Added Entry. Following implementation of the LCGFT for music, genre/form information for music will be recorded in the MARC 655 field, Index Term-Genre/ Form. Libraries and vendors may have addressed treatment of genre headings in discovery tools in response to the implementation of other LCGFT projects, such as moving images. Faceted interfaces may also treat form subdivisions in subject headings ( $\$ \mathrm{v})$ as genres. 
Since genre headings are already in use, it is easy to evaluate their indexing, display, facets, and linking functionality in a database that contains those headings. Recently cataloged video recordings are likely to include genre headings, and some libraries may have made retroactive changes to older records. A known-title search will reveal if headings appear under a genre facet and how headings display and function in individual records. A search for a genre term that is not also used in LCSH, such as \{"classical music radio programs"\}, will help in evaluating indexing and facets. Some genre/form terms contain elements that overlap with LCSH topical headings ("opera films"). Terms with identical forms in both LCGFT and LCSH ("field recordings") may be useful as searches to determine whether the term appears more than once under a single facet or under multiple facets.

\section{Medium of Performance}

Medium of performance is currently recorded in controlled form in subject headings or in codes in an optional MARC 048 field, used only for music materials. RDA records may also record medium in the MARC 382 field, Medium of Performance. Following the implementation of the LCGFT and LCMPT, the medium of performance terms currently part of LCSH and recorded in the MARC 650 field will be recorded in the MARC 382 field in recognition that they are not genre/form or subject terms. ${ }^{24}$ The eventual widespread use of the 382 field means that a medium index may be possible in discovery tools. Testing the treatment of the 382 field will require a critical mass of records that contain the field. The LCMPT has not yet been implemented.

\section{Physical Description Statements}

Because music materials may be issued in multiple pieces or formats, physical description statements (MARC 300) may be more complex than for books. Discovery tools differ in display of subfields, particularly those following the first occurrence of $\$$ a. Scores and parts issued together are all of primary importance and are described with separate terms in subfield $\$ \mathrm{a}$; parts are not considered or coded as accompanying material in $\$ \mathrm{e}$. In some cases, primary materials may be of different dimensions, so the field becomes more complex, with subfields for extent $(\$ \mathrm{a})$ and dimensions $(\$ \mathrm{c})$ repeated. ${ }^{25}$ Display in a public interface is likely of more use than indexing, since format facets/limits or subject subdivisions permit users to select format. To locate records to evaluate display of complex 300 fields it may be necessary to perform intermediate searches using a staff utility such as a cataloging module within an ILS if the field is indexed, or OCLC Connexion, which offers an index that searches extent $(300 \$ \mathrm{a})$. Alternatively, one may search for known items.
Media that accompanies print formats may be indicated in a repeated MARC 300 field in RDA records or in subfield $\$$ e (accompanying material) in a 300 field in RDA or AACR2 records, and music catalogers will likely continue to use $\$ \mathrm{e}$ for recordings accompanying books or scores. ${ }^{26}$ The Hal Leonard Play-Along and Jamey Aebersold Jazz series are issued as lead sheets (notated music) with accompanying CDs. These are usually cataloged on a single record with the score as the primary format ( $300 \$ \mathrm{a} \$ \mathrm{~b} \$ \mathrm{c})$ and the CD as accompanying material $(300 \$ \mathrm{e})$. These series and other score and CD sets usually contain series titles, subject headings, or notes that can be combined to identify records. Most music titles with an additional format indicated in \$e will also include appropriate added 006 and 007 fields that can be used for limits, facets, icons, or labels.

\section{Format Options}

Format is a general term that does not correspond exactly to RDA content, carrier, or media types. Specific format terms used in discovery tools may combine characteristics of content, carrier, and media. Different products and different libraries' implementations of the same discovery tool may employ different terms and definitions. MARC leader (LDR) codes, at times combined with codes in fields 006 and 007 , allow mapping to many precise formats that can be used in limits, facets, labels, or icons. Identifiable formats, which may be labeled with various terms, include book, cassette tape, compact disc, DVD, LP, manuscript, music recording, score, sound recording, spoken word recording, and video recording. MARC coding is sufficiently precise to identify additional formats, including $78 \mathrm{rpm}$ discs, negative disc stampers, or wax cylinders, that could be of interest for specialized collections. ${ }^{27}$ Libraries may wish to customize format options and labels to best reflect their collections and meet users' needs.

Format facets and icons may be explored with a blank or broad search. Searching a known item and reviewing resulting icons, labels, or facets can also indicate completeness and accuracy of indexing. Considerations include the following: Does a limit or facet selection of a format lead to results that include all properly coded records for that format? Are additional formats included that are not implied by the term? With scores, incomplete results can occur with the format option "manuscript," which is often mapped only to text (LDR/06 " $\mathrm{t}$ "), even though a code is available to indicate manuscript music (LDR/06 "d"). To determine whether music is included in the "manuscript" format, one may search for a known music manuscript and look for results with "manuscript" as a format facet option, label, or icon. A subject or keyword search for \{manuscript or \{manuscripts\} will not be accurate because "manuscripts" is used in subject headings, and "manuscript" is a common 
keyword. In other cases, formats retrieved may be broader than a term implies. A discovery tool may offer "moving image" as a format option, but items like slides or filmstrips may also be included if a broader mapping is used (LDR/06 "g" Projected Medium). Testing for instances like this can be difficult, and may best be accomplished with a search for a known item.

For those records where multiple formats are reflected in a single record, searches and examples similar to those for physical description/accompanying materials will also test facets or icons, provided the associated MARC 006 (additional material characteristics) or 007 (physical description) fixed fields are present in bibliographic records. A search for a known title issued with accompanying material of a different format will indicate if added formats are offered in facet options or represented with icons or labels in brief results or individual records. For the same title, a pre-search limit for the accompanying format will indicate whether that format is indexed. Searches for the customary AACR2 note phrase \{ "accompanying compact disc"\} should indicate the relative numbers of print materials and recordings under a format facet.

Content, media, and carrier (MARC fields 336-338) will be present in all RDA records. Assuming a library retains these fields in its local catalog, any RDA record can be used to evaluate display. Terms in the vocabularies for each are not necessarily unique to those fields, so knownitem searches may be best to use to determining indexing. The number of records containing the MARC 344-347 fields (sound, moving image, video, and digital file characteristics) should increase as RDA is gradually adopted. One may use terms in the relevant RDA instructions in chapters 3.16-3.19 as search terms to retrieve records to evaluate display and explore indexing. If indexing of these fields in a discovery tool has not yet been defined, terms in these fields are indexed as entity attributes in OCLC Connexion and FirstSearch and as general keywords in the freely available WorldCat.org. ${ }^{28}$

\section{Language Limits, Facet, or Labels}

Bibliographic records for instrumental music contain the MARC language code for "no linguistic content" ("zxx"); this code is also used for models, realia, and other items that do not provide language characteristics. Limits, displays, and language facet options vary among discovery tools. Terms generated from "zxx" ("music," "no language," or "no linguistic content") might be offered as language choices in an advanced search. A search for orchestral music or symphonies narrowed to scores should allow sufficient expansion of a language facet to determine whether a term representing code "zxx" appears, or a record for an instrumental work can be examined to determine whether a term is included. The code is only used if all works in a compilation lack linguistic content, so a limit to scores increases the likelihood of retrieving instrumental-only works.

For items with multiple primary languages, there is no coding distinction between a single work in multiple languages and a compilation of works each in different languages. Records likely to contain codes for multiple primary languages (041 $\$ \mathrm{a}$ or $\$ \mathrm{~d}$ ) include compilation sound recordings of vocal works, particularly if they are compiled based on performer rather than composer, and score anthologies of arias or art songs. When testing items with multiple languages coded in the same subfield, the MARC record should be checked for current practice, which uses the granular subfield coding that some systems require to identify languages (041 \$a eng \$a fre \$a ger instead of the older \$a engfreger).

If searching for translations of musical material, vocal scores of operas with parallel texts are among the most common instances of musical materials with both the original language and a translation. Uniform titles in AACR2 may employ "polyglot" to indicate three or more languages, representing at least two translations, making the search \{polyglot scores\} useful until the term-obsolete in RDA-is no longer represented in a sufficient number of catalog records for an effective search. Subtitles in the language sung and translations are standard in opera DVDs. A video recording or "visual material" search for \{operas subtitles\} or \{filmed operas subtitles\} should be sufficient to yield results that indicate how subtitles are treated.

For the purposes of coding language content, even written material included in a music resource may be considered "accompanying material." Scores frequently contain editor's introductions or written performance instructions, but only the language of vocal text within the notated music itself is considered "primary." Searches for sound recordings (limit/ facet) are likely to locate records containing codes for accompanying materials in one or more languages. Many commercially issued sound recordings are issued with program notes that include multiple translations or with printed vocal texts and translations. A search designed to retrieve records for instrumental music (without linguistic content) that retrieves numerous records labeled as being in a language such as English indicates that accompanying materials are indexed as a language. Records for scores often refer to "critical commentary" or a "critical report," and some series are issued with extensive introductory material or editorial notes. Searches such as \{critical commentary scores\} should retrieve records for scores with significant accompanying material.

\section{Notes}

Notes relevant to music materials include those for language or notation, recording information, production credits, performers, and contents; general notes also contain important 
information. Most notes contain information needed for users to identify or select a resource, and any suppression of notes fields should be a deliberate choice.

\section{Language or Notation Note}

AACR2 records for music resources frequently include a dedicated language note (MARC 546) indicating language of sung or spoken text for vocal works. RDA records for scores also indicate musical notation in this field ( $546 \$ \mathrm{~b}$, Information Code or Alphabet). The increased use of this subfield in music cataloging means indexing and display must be tested even if language notes have been displayed and indexed satisfactorily in the past. Records for scores of vocal works will include both subfields $\$ \mathrm{a}$ and $\$ \mathrm{~b}$ in one or more 546 notes. Records for notated instrumental music may include $\$$ b only. Keyword searches for \{"staff notation"\} or \{“graphic notation"\} may not test indexing of this subfield because these phrases are routinely recorded in general (500) notes in AACR2 records. Currently, field 546 cannot be searched in any of the WorldCat indexes as an intermediate step to identify records. ${ }^{29}$ It may be easiest to identify RDA records for notated instrumental music, then search based on known data in the record.

\section{Performer and Cast Information Note}

Performer and cast information is recorded in a dedicated note (MARC 511). Although performer and cast names can appear elsewhere in a record, this may be the only place where performers are associated with particular dramatic roles or instruments. It may also be the single place where their names match conventional usage. Determining whether the note displays and if the label differs for each indicator (5110\#, No Display Constant Generated, customarily used with music sound recordings, or 5111\#, Cast, customarily used with general feature films) can easily be accomplished by comparing records for feature films and musical sound recordings.

Testing inclusion of the MARC 511 field in an author keyword index through searches requires identifying records where the form of a name transcribed in the 511 field does not appear in any other potential "author" fields (1xx, 245 $\$ c, 505,508,7 \mathrm{xx}, 8 \mathrm{xx})$. This may occur most frequently where the form of a performing group's name is in English (e.g., "Vienna Philharmonic") in the note but in another language ("Wiener Philharmoniker") in the authorized access point. An alternate search method is to include non-name information from a 511 note in an author-index search, avoiding terms that may be present in other "author" fields within the same record.

\section{Production Credits Note}

Creation and production credits for creators and contributors other than performers or cast are recorded in a dedicated note (MARC 508). This is routinely used in records for video recordings, where it can contain names of creators or contributors related to music including conductors and composers. Using searches to test whether information in this note is contained in an author index is problematic because the names may also be represented in access points. It may be possible to search for roles in an author keyword index: \{producer director choreographer\}. However, as relator terms appear more frequently with name access points in RDA records, it will be difficult to use a search to distinguish between indexed subfields $\$ \mathrm{e}$ in name headings and indexing of field 508 .

\section{Recording Information Note}

Recording information for music sound and video recordings is provided in a dedicated note, MARC 518 field, Date/ Time and Place of an Event. In addition to date and place information, this note may also indicate whether the recording was live, the names of specific venues, or distinctions of place or date by work in a compilation. Notes in AACR2 records utilize a single subfield $(\$ \mathrm{a})$ with easily readable contents. More granular subfields with RDA mean that subfield-specific labels may be required to make the note easy for a user to interpret. These notes lack subfield $\$$ a and record date, place, and other information in subfields $\$ \mathrm{~d}, \$ \mathrm{p}$, and $\$$ o, respectively; the field may be repeated with distinction by work in subfield $\$ 3$. Because use of these subfields is new, a review of display, labeling, and indexing of the field and subfields may be desirable even if the field was indexed and displayed according to a library's preference in the past. To test indexing of the MARC 518 subfields $\$ \mathrm{~d}, \$ \mathrm{p}$, and $\$ \mathrm{o}$ through a search, content in any of these subfields that is not present elsewhere in a retrieved record can be entered as a search together with composer or performer name.

\section{Contents Note}

Formatted contents notes (MARC 505) contain useful, detailed, and eye-readable information about titles, composers, performers, and other contributors. Analytic access points are not typically used for song titles in records for popular music materials. Cataloging rules or practicality may preclude comprehensive analytic added access points (7xx) for musical works in compilations. Furthermore, the contents note may be the sole field where the title in the bibliographic record matches the title on the item.

To test author or title indexing of this note, it is necessary to identify records with contents notes that contain 
contributor and title information not present in fields potentially indexed as author or title (1xx, 24x, 508,511,7xx, 8xx). These tend to be compilations or anthologies with a large number of works or recordings of popular music. When items are identified, a search in an author keyword index for data present only in $\$ \mathrm{r}$ (enhanced) or $\$ \mathrm{a}$ (non-enhanced) and a name from a 100 or 700 field will indicate if information in $\$ \mathrm{r}$ is an author index. To determine whether $\$ \mathrm{t}$ is in a title index, the same process can be repeated with a title from $\$ \mathrm{t}$ and terms from the title proper $(245 \$ \mathrm{a})$.

\section{Edition Information}

Edition information for music materials recorded in the MARC 250 field, Edition Statement, may provide significant information, including the editor of the music or transcribed information about vocal range. In RDA, edition information recorded in this field also includes details related to arrangement, currently recorded in the statement of responsibility (245 \$c), or related to score format, previously recorded in the 254 field, Musical Presentation Statement, obsolete in RDA. ${ }^{30}$ Both fields will coexist in a database that contains AACR2 and RDA records.

The potential redundancy of terms in the MARC 250 and 254 fields with information elsewhere in a record makes it difficult to use a search to differentiate types of indexing. Some discovery tools may not index the musical presentation statement, making searching within this field impossible. Currently, the 254 field cannot be searched in any of the WorldCat indexes. ${ }^{31}$ Display may best be evaluated using records known to contain these fields.

\section{Publisher, Music, or Plate Numbers}

Music materials typically lack ISBNs, and may include publisher, issue, or plate numbers. Publishers' numbers for scores and issue numbers for recordings are used similarly to ISBNs for ordering, searching, and identification. They are recorded in the MARC 028 field, Publisher Number. Discovery tools offer different capabilities related to the indexing of numbers in $028 \$ \mathrm{a}$ and the indexing of the publisher in $028 \$ \mathrm{~b}$. Bibliographic records for recordings and publications by jazz label Mosaic Records and classical score reprint publisher Recital Publications frequently include multiple occurrences of the 028 field, recording both original and reissue or reprint information in subfields $\$ \mathrm{a}$ and $\$ \mathrm{~b}$. Determining indexing of numbers in the 028 field requires a publisher number index and a known item; CD box sets are typically suitable. The display of subfield $\$ \mathrm{q}$, Qualifying Information, defined in 2012 , may evaluated by identifying newer records for music resources, particularly CD boxed sets, that contain the subfield.

\section{Diacritics, Special Characters, and Stopwords}

Musical sharps and flats are essential to the musical key element in preferred titles and may be present in transcribed titles. ${ }^{32}$ Diacritics are common in records for music materials, which frequently include works, imprints, and access points in languages other than English. How all of these are searched and displayed is important. There are abundant instances of access points that contain sharps, flats, and diacritics. Searching using the flat $(b)$ and sharp (\#) symbols may require copying the symbols from another source. It is possible that the pound sign (\#) functions as a sharp sign, but it should be noted that some discovery tools use the symbol (\#) as a wildcard character. ${ }^{33}$ Search capability can be explored with pairs or sets of searches using the correct symbol, the equivalent word, and (for sharps) the pound sign. Czech, German, and Russian names and titles frequently include many diacritics; special characters (i.e., degree signs and superscripts) are not common in music records but can appear in titles of contemporary works. Evaluation using known items may be the most effective strategy in such cases.

RDA introduces a new display concern for sound recordings. Instructions on recording copyright dates require a phonogram date to be preceded by the phonogram symbol (®) if it can be reproduced when the data are recorded. ${ }^{34}$ This is a change from AACR2 practice where the symbol was recorded as "p." The phonogram or copyright symbols may display incorrectly when a system is unable to accommodate them. The phonogram symbol is common in sound recording records, and display can be determined by retrieving an RDA record that contains the symbol.

Usually a stopword, "a" is meaningful when part of musical key. Without a way to force "a" into a phrase search, users searching for a musical form plus \{“A major"\} (intending the musical key A major) will retrieve other terms with "major," leading to erroneously broad results for musical works (musical keys C major, D major, A major, etc.). Another common element of transcribed and preferred titles that needs to be searched as a meaningful term is "no." (abbreviation for number), which may function as a Boolean operator in some tools. Testing of phrases versus stopwords or operators requires only a search for the same terms with and without quotes, followed by a comparison of the number of results.

\section{Conclusion}

The scope of this paper is narrow within the world of discovery tools. Details such as the display of specific notes fields and indexing of publisher numbers from MARC records are critical for the discovery of music materials, but many more 
resources are represented by AACR2 and RDA MARC bibliographic data. Thorough evaluation of discovery tools will require further steps. There are other types of resources that contain complex or specialized bibliographic description, such as rare books, serials, or archival collections, which can be used similarly to music for evaluating a discovery tool. With web-scale tools, it is essential to evaluate index, display, and linking for content in the entire central index, which encompasses more than AACR2 and RDA MARC records. Records created from sources within and outside library holdings with different descriptive and coding standards raise issues including metadata harmonization and de-duplication.

Looking ahead, discovery tools will develop new features and functionalities beyond those related to flat bibliographic records. The utilization of authority data and the relationships integral to FRBR and RDA have not been fully realized in discovery tools. The BIBFRAME model and linked data also offer opportunities for adding new functionality to discovery tools. As discovery tools and services emerge and develop new capabilities, and users' expectations demand continual improvement, the need for evaluation will only increase.

An awareness of the data being used and of the metadata schemes and descriptive rules in use, a checklist of questions, and a supply of examples and strategies together create a flexible and reusable evaluation method that is a valuable component of a comprehensive assessment of discovery tools. Catalogers and librarians who are aware of the capabilities of the data available to discovery tools must play an ongoing, integral role in ensuring that discovery tools go beyond meeting basic user needs and realize the full potential offered by the data itself.

\section{References and Notes}

1. Athena Hoeppner, "The Ins and Outs of Evaluating WebScale Discovery Services," Computers in Libraries 32, no. 3 (2012): 7.

2. Priscilla Caplan, "On Discovery Tools, OPACs and the Motion of Library Language," Library Hi Tech 30, no. 1 (2012): 108-15.

3. Geoffrey Little, "Thinking about Discovery Layers," Journal of Academic Librarianship 38, no. 6 (November 2012): 346.

4. VuFind (http://vufind.org) is in use at Villanova University (http://library.villanova.edu). Blacklight (http://projectblacklight.org) is in use at Stanford University (http://searchworks. stanford.edu) and has an optional music interface at the University of Virginia (http://search.lib.virginia.edu/music). BiblioCore (www.bibliocommons.com/products/bibliocore) is in use at the New York Public Library (http://nypl.bibliocommons.com). Summon (www.serialssolutions.com/en/services/ summon) is in use at North Carolina State University (www. lib.ncsu.edu). An example of Primo (www.exlibrisgroup. com/category/PrimoOverview) is at Northwestern University (www.library.northwestern.edu). WorldCat Local (www. oclc.org/en-US/worldcat-local.html) is in use at the University of Maryland (www.lib.umd.edu). For additional information on the new FirstSearch that will eventually replace the current WorldCat Local and FirstSearch WorldCat interfaces, see "What to Expect in the New User Experience for FirstSearch," OCLC, accessed July 30, 2013, www.oclc.org/go/en/ firstsearch-migration/expect.html.

5. Hoeppner, "Evaluating Web-Scale Discovery Services," 8. A slightly longer list is provided in Sharon Q. Yang and Kurt Wagner, "Evaluating and Comparing Discovery Tools: How Close Are We towards Next Generation Catalog?" Library Hi Tech 28, no. 4 (2010): 693-95.

6. The discovery tool VuFind offers a browse search and may be developed to make use of authority data; for a more detailed discussion, see Demian Katz, Ralph LeVan, and Ya’aqov Ziso, "Using Authority Data in VuFind," Code4Lib 14 (July 2011), accessed July 30, 2013, http://journal.code4lib.org/articles/5354.

7. The existence of multiple manifestations per work is evident in a WorldCat search for a major work in the canon of Western art music. For an early, quantitative study confirming the widespread occurrence of multiple manifestations, see Richard P. Smiraglia, "Uniform Titles for Music: An Exercise in Collocating Works," Cataloging \& Classification Quarterly 9, no. 3 (1989): 97-114.

8. Music Library Association, Emerging Technologies and Services Committee, "Music Discovery Requirements," 2012, accessed October 28, 2013, http://committees.musiclibraryassoc.org/uploads/ETSC/MDRdocument.pdf.

9. Ibid., 2 .

10. "Bibliographic Framework Transition Initiative," Library of Congress, accessed December 6, 2013, www.loc.gov/marc/ transition.

11. "Genre/Form Terms for Musical Works and Medium of Performance Thesaurus," Library of Congress, accessed July 30, 2013, www.loc.gov/catdir/cpso/genremusic.html.

12. Victoria O'Rourke, "Selection of an Online Public Access Catalog: A Checklist Approach," Information Technology \& Libraries 6, no. 4 (December 1987): 282; the checklist is reproduced on pages 285-87.

13. Truong Dai Luong and Chern Li Liew, "The Evaluation of New Zealand Academic Library OPACs: A Checklist Approach," Electronic Library 27, no. 3 (2009): 376-96; tables appear on pages 380-388.

14. Jason Vaughan, "Investigations into Library Web-Scale Discovery Services," Information Technology \& Libraries 31, no. 1 (March 2012): 56-58.

15. Malcolm Ramsay and Edmund Chamberlain, "Software Selection Methodology for Library Discovery Layer Systems," FOSS4Lib, accessed July 30, 2013, http://foss4lib.org/ 
decision-support/discovery-layer-ssm.

16. Yang and Wagner, "Evaluating Discovery Tools," 690-709.

17. Nara L. Newcomer, "Summon," Music Reference Services Quarterly 14, no. 1-2 (2011): 59-62; Rebecca Belford, "WorldCat Local Enhancement Recommendations for Music," Music OCLC Users Group Newsletter 111 (September 2012): 14-18; Lisa Hooper, "Ex Libris's Primo and Musical Research," Music Reference Services Quarterly 15, no. 2 (2012): 119-24.

18. Jody Condit Fagan, "Usability Studies of Faceted Browsing: A Literature Review," Information Technology \& Libraries 29, no. 2 (2013): 58-66.

19. Tracey Snyder, "Music Materials in a Faceted Catalog: Interviews with Faculty and Graduate Students," Music Reference Services Quarterly 13, no. 3-4 (2010): 66-95.

20. Verletta Kern, "FirstSearch Advisory Group" (presentation at the annual meeting of the Music OCLC Users Group, San Jose, California, February 27, 2013), accessed July 30, 2013, www.musicoclcusers.org/mougmeetings/2013meeting/FirstSearch\%20Advisory\%20Group.pptx.

21. Terry Ballard, "Comparison of User Search Behaviors with Classic Online Catalogs and Discovery Platforms," Charleston Advisor 12, no. 3 (January 2011): 65-66; Kelly Meadow and James Meadow, "Search Query Quality and Web-Scale Discovery: A Qualitative and Quantitative Analysis," College \& Undergraduate Libraries 19, no. 2-4 (2013): 163-75.

22. Many of the access points included as examples in appendix $\mathrm{B}$ were provided to programmers at the author's institution in response to requests for MARC examples related to system enhancements in an open-source discovery layer.

23. The instruction sheets that govern form subdivisions for notated music in the Subject Headings Manual are H 1160 (musical compositions) and H 1161 (musical instruments). Library of Congress Cataloging Distribution Service, Subject Headings Manual, 1st ed. (Washington, DC: Library of Congress, 2008).

24. MARC Proposal No. 2012-01, "New Data Elements in the MARC 21 Bibliographic and Authority Formats for Medium of Performance," accessed July 30, 2013, www.loc.gov/marc/ marbi/2012/2012-01.html.

25. This continuation of this practice from AACR2 to RDA is affirmed in the recommended application of the exception for notated music to RDA 3.5.1.6. Music Library Association, Bibliographic Control Committee, RDA Music Implementation Task Force, "Best Practices for Music Cataloging Using
RDA and MARC21," draft, February 15, 2013, accessed July 24, 2013, http://bcc.musiclibraryassoc.org/BCC-Historical/ BCC2013/RDA_Best_Practices_for_Music_Cataloging.pdf.

26. Music Library Association, Bibliographic Control Committee, RDA Music Implementation Task Force, "Best Practices for Music Cataloging," 31.

27. The degree of specificity of identifiable formats can be seen in "MARC 21 Format for Bibliographic Data," particularly the 007-Physical Description Fixed Field, accessed July 24, 2013, www.loc.gov/marc/bibliographic/bd007.html, and in "Searching WorldCat Indexes: Format and Material Type Values Indexed," OCLC, accessed July 30, 2013, www.oclc.org/ support/services/worldcat/documentation/searching/searchworldcatindexes.en.html\#search_worldcat_materialtypes.fm.

28. "Searching WorldCat Indexes," OCLC, accessed July 30, 2013, www.oclc.org/support/services/worldcat/documentation/searching/searchworldcatindexes.en.html; confirmed by searches in Connexion Client and WorldCat Local, July 30, 2013.

29. "Searching WorldCat Indexes"; confirmed by searches in Connexion Client and WorldCat Local, July 30, 2013.

30. RDA: Resource Description and Access 2.5.2.1 (g) includes "a particular voice range or format for notated music" within the scope of "edition." In the examples following in 2.5.2.2, both "Full score," which in AACR2 would be considered a musical presentation statement, and "Vollständiger Klavierauszug," which would be considered part of a statement of responsibility, are included as examples of designation of edition. RDA: Resource Description \& Access (Chicago: ALA; Ottawa: Canadian Library Association; London: Chartered Institute of Library and Information Professionals, 2010), accessed July 30, 2013, http://access.rdatoolkit.org.

31. "Searching WorldCat Indexes."

32. For more about catalog issues related to flats and sharps, see Barbara Henigman and Richard D. Burbank, "Online Music Symbol Retrieval from the Access Angle," Information Technology \& Libraries 14, no. 1 (1995): 5-16.

33. The sign is used as a wildcard character in the WorldCat.org, WorldCat Local, and FirstSearch WorldCat interfaces."WorldCat Help," OCLC, accessed July 20, 2013, www.oclc.org/support/help/worldcatorg/ApplicationHelp. htm; "Searching WorldCat Indexes."

34. RDA, 2.11.1.3: Recording Copyright Dates.

\section{Appendix A. Checklist Questions for Music-Focused Discovery Tool Assessment}

This appendix is a checklist for evaluating treatment and presentation of AACR2 and RDA MARC records in a discovery tool. It is not a comprehensive checklist, but does include general features that have an impact on searching for music resources. The list includes sections exclusive to music, which are clearly identified. All field tags, indicators, and subfields refer to the MARC 21 format.

Many questions are open ended to provide the opportunity for a library to consider its own desired functionality; for yes/ 
no questions, a "yes" answer does not necessarily represent desired functionality. For indexing and display recommendations related specifically to music, readers may wish to consult the music discovery resources available from the Music Library Association.

\section{General Questions}

- Which MARC fields and subfields display?

- How are displayed fields and subfields labeled?

- Are field tags, indicators, or subfields used to generate specific labels?

- Which fields display (1xx through $8 \mathrm{xx}$ )? In what order? How are they grouped?

- Is any coded information (LDR/0xx) indexed or displayed in a readable form for the end user?

- How does subfield order within a field compare to the original MARC record?

- Which indexes are available for searching? Which fields/subfields are included in each index?

- Which indexed information is visible in a record?

- Are search terms visible in brief results or single records?

- What information is used to determine facets or limits? How does this compare to search indexes?

- What display, index, and ranking options are customizable?

\section{Headings (controlled subjects and genre/form terms, names, titles)}

- Where in the record do headings display? How are they grouped?

- Are headings clickable links?

○ Which subfields within a heading are linked?

$\circ$ Are subfields bound together as a single link?

- Do linked headings generate a keyword search for each term or subfield separately (Boolean AND) without regard to order?

- In which indexes do linked headings generate a search?

- Is there an option to link to a user-selected portion of a heading to execute broader or narrower searches (decreasing precision by clicking on subfields from right to left in an access point)?

- Is browse searching available?

- How should terms be entered?

- Do terms need to be entered in a specific order?

- How does a browse search function when terms other than authorized forms of names, titles, or subject/genre headings are entered? Does it make a difference if the terms entered are see references in authority records?

- Do clickable links on headings generate browse searches?

- What use does the tool make of authority records?

\section{Names}

- How and where does the statement of responsibility (245 \$c) display in relationship to the title in the 245 field and names in $1 \mathrm{xx} / 7 \mathrm{xx}$ fields?

- Which fields containing names display in brief results (information from 1xx, 7xx, $245 \$ \mathrm{c}, 508,511$, other)? Is this dependent on format? For example, lxx for books but 511 for sound recordings.

- Do elements within $\$$ a in name access points display in the desired order?

- Are name access points displayed fully enough for identification and differentiation?

- Which name access points or portions of name access points appear under an author facet?

- Corporate or conference names as main entry $(110,111)$ ?

- Names in added entries $(700,710,711)$ ?

- Names in series $(800,810,811)$ ?

- Names in added entries that contain title $(\$ t)$ subfields?

- In single records, are there label or display distinctions between main and added author access points (1xx versus 7xx, 8xx)? Between personal, corporate, conference, or series names?

- Do names in subject fields $(600,610,611)$ appear under a subject facet? 


\section{Titles of Works and Expressions}

- Are preferred titles (130, 240, 7xx $\$$ t with additions, 730) identified or indexed separately from other title access points $(245,246,740)$ ?

- Is there a separate, additional index for preferred title?

- Are all subfields within a preferred title field linked as a single (bound) search string?

- If access points for preferred titles include names:

- Do titles in field 240 display with or near the associated name in a 1xx name access point entry?

o Is a link in field 240 bound to the name in a $1 \mathrm{xx}$ name access point (name-title search)?

- Are links on titles in name-title added entries bound to their associated names?

$\circ$ How is a title in a name-title access point displayed in relationship to its associated name? In other words, is it clear who wrote what work?

- If a record contains both name and name-title added entries, how are headings of each type grouped? Does the presence of title subfield $(\$ \mathrm{t})$ prompt headings in $7 \mathrm{xx}$ name fields to be labeled differently than name headings without titles? For example, "Contributor" versus some sort of label suggesting a work or title.

- Do searches or browses for a numbered work within a number range in a title subfield $\$$ n retrieve records where only the first and last numbers in the range are recorded?

\section{Analytic Entries}

- Do analytic title entries (7xx with 2nd indicator " 2 ") display?

- Are controlled analytic entries (titles in MARC fields 700, 710, 711, 730) differentiated in display, linking, or indexing from uncontrolled analytic entries (740)?

- Are indicators in MARC 7xx name or title fields used to generate a display? For example, "Contains" or "Related title."

\section{Relationships}

- Are relator codes $(\$ 4)$ or relationship designators $(\$ \mathrm{e})$ displayed?

- Are codes displayed in a spelled-out form ("performer" versus "prf")?

- Is information in relator codes or terms used for additional indexes or facets (performer, conductor, composer, editor, etc.)?

- Does information in 7xx \$i display? Does all information in \$i display? ("Based on (work)" versus "Based on," etc.)?

- Does the presence of $\$ \mathrm{i}$ in a $7 \mathrm{xx}$ field suppress a general $7 \mathrm{xx}$ field label?

- How is data in 7xx $\$ \mathrm{i}$ handled in browse, keyword, name, or title indexes?

- Is there any linking functionality based on the $R D A$ relationships indicated in a subfield $\$ \mathrm{i}$ (navigation to all parodies, or all works based on a particular play, etc.)?

\section{Subjects}

- Do title headings in subject fields (630,600 \$t and subfields) appear under a subject facet? With associated names?

- Are non-topical subject subdivisions $(\$ \mathrm{v} \$ \mathrm{y} \$ \mathrm{z})$ distributed over facets other than subject?

\section{Genre/Form}

- Is there a genre facet?

- Is there a genre index? Which fields or subfields are included?

- Are genres indexed as subjects?

- Is there an option to search a combined subject/genre index?

- Are form subdivisions $(\$ \mathrm{v})$ in subject headings indexed as genres or included under a genre facet?

- How is related coded information for music genre/form (008/18-19, 047) used? 


\section{Medium of Performance (music only)}

- Are medium of performance fields (382, \$m within title fields 130, 240, 6xx, 7xx) indexed and displayed?

- Is there a separate, additional index for medium?

- Is a customized search interface for medium available?

- How is coded medium information (048) used?

\section{Format Limits, Facets, Icons, and Labels}

- What icons, labels, and terms are used?

- What formats are offered in limits/facets? Can multiple formats be selected to expand search results?

- Is there sufficient specificity?

- Are labels logical, precise, and accurate? Do labels reflect content, carrier, media, or a combination?

- Can format(s) be easily identified in brief results and in individual records? How?

- Are multiple formats coded in a single record each indexed and displayed?

- How is the general material designation $(245 \$ \mathrm{~h})$ used if present?

- How is data in 33x or 34x fields utilized to indicate format or provide facets/limits?

\section{Language Limits, Facet, or Labels}

- Does the code for "no linguistic content" (zxx) generate a limit, facet option, or label? What term is used?

- How is coded language information (008/35-37, $041 \$ \mathrm{a}$, \$d) indexed, displayed, or used for limits/facets?

- Are codes for subtitles $(041 \mathrm{\$ j}$ ) treated as a primary language for indexing and limits/facets?

- How is coded information about translations (041 1st indicator, $041 \$ \mathrm{~h}, \$ \mathrm{k}, \$ \mathrm{~m}, \$ \mathrm{n})$ used?

- Is coded information for accompanying material $(041 \$ \mathrm{~b}, \$ \mathrm{e}, \$ \mathrm{f}, \$ \mathrm{~g})$ or subtitles $(041 \$ \mathrm{j})$ indexed, displayed, or used for limits/facets? Are there more detailed facets based on the subfield definitions in 041?

- How is field 377 (associated language) indexed or displayed?

\section{All Notes $(5 x x)$}

- Which notes fields display? Where do they appear in single records?

- Do repeatable fields display?

- In what order do notes appear (MARC record order, MARC numeric tag order, custom order)?

- Are notes distributed among multiple tabs or areas of a bibliographic record?

- How are notes indexed? Are notes or subfields included in multiple indexes?

\section{Language/Notation Note (546)}

- How is subfield $\$$ b indexed or labeled?

- How is related coded information (008/35-37, 041) used?

\section{Performer/Cast Information Note (511)}

- Does information appear instead of or with statement of responsibility $(245 \$ \mathrm{c})$ in brief results or single records?

- Is information in this note also indexed in an author index?

\section{Recording Information Note (518)}

- How are subfields $\$ \mathrm{~d}, \$ \mathrm{p}$, and $\$$ o indexed, displayed, or labeled? Does information in $\$ 3$ display?

- How is related coded information (033) used? 


\section{Formatted Contents Note (505)}

- Is the entire field for non-enhanced notes $(\$ a)$ included in author or title indexes?

- Are different subfields in enhanced contents notes indexed in appropriate indexes in addition to keyword: author index $(\$ r)$ and title index $(\$ t)$ ? Is information in $\$ g$ indexed?

- Is punctuation within contents notes used to generate line breaks or layout?

\section{Edition Information (254) (music only)}

- Is any distinction in indexing or display made between fields 250 (edition) and 254 (musical presentation)?

\section{Publisher, Music, or Plate Numbers (028) (music emphasis)}

- Is there a separate, additional index for music number $(028 \$ \mathrm{a})$ ?

- Is the publisher information in $028 \$$ b included in a publisher index?

- Do searches or browses for a publisher number within a consecutive range retrieve records where only the first and last numbers in the range are recorded in $028 \$ \mathrm{a}$ ?

\section{Diacritics, Special Characters, and Stop Words (music emphasis)}

- Do diacritics, sharps, flats, special characters, and the phonogram/copyright symbols display properly?

- Do the words "sharp" or "flat" generate searches for the respective symbols?

- Is there a way to enter sharp and flat signs from the search interface?

- Does the pound sign (\#) function as a sharp sign?

- Does the pound sign (\#) function as a wildcard operator?

- Do symbols pasted into a search function as those symbols?

- Does retrieval of records with diacritics require searches to be entered with diacritics?

- Is phrase searching possible?

- Can potential stopwords such as "a" or potential Boolean operators such as "no" be forced to function as search terms if enclosed in quotation marks?

\section{Appendix B. Sample Searches, Authorized Access Points, and Titles for Music-Focused Discovery Tool Assessment}

Four types of examples follow: searches, WorldCat command searches, authorized access points, and titles with excerpts of bibliographic records from WorldCat.

\section{Searches}

Searches may be conducted in a discovery tool to test indexing, to retrieve a set of results to explore facets and brief results, or to retrieve records to investigate display and linking functionality. Curly brackets [ \{\}$]$ surround single searches, and terms within a single search are assumed to be combined (Boolean AND). Any quotation marks included within brackets are intended as part of the search query. Searches without any index indicated are assumed to be general keyword searches. Searches are assumed not to be case-sensitive; titles within search strings are not capitalized.

\section{WorldCat Index Command Searches}

In a few cases, WorldCat index command searches for use with OCLC's products that share indexes (Connexion, Cat Express, FirstSearch, WorldShare, WorldCat Resource Sharing) are included, limited to cases where a discovery tool is unlikely to index the affected fields or where there are insufficient examples in WorldCat at the time of writing. These searches can help identify resources held by a library that can then be searched in a local system. Examples are shown with an optional limit by holding library, with DLC (the symbol for Library of Congress in OCLC) used as an example. 


\section{Authorized Access Points}

Authorized access points may be used as a basis for searches. These are given with full MARC coding, which may be useful for back-end customization or comparison with public record views. Authorized access points should be compared against the authority file to ensure that the most recent form is being used.

\section{Titles with Excerpts of Bibliographic Records from WorldCat}

Titles and excerpts of bibliographic records from OCLC's WorldCat database illustrate various topics. Examples were selected based on a relatively large number of holding institutions in WorldCat and accuracy in coding of the field(s) in question; titles demonstrating multiple topics were given preference. The format is indicated, followed by title and OCLC accession number. Relevant MARC fields are reproduced below the title. MARC field tags and subfield delimiters are retained in examples when needed for comparison with local catalogs or for internal systems use, with indicators included only if necessary, with “\#” for blanks.

The topics illustrated by each set of examples are identified, followed by the type of example.

Names. Corporate names contained in access points. Searches:

\{Beatles\}

\{Boston Pops Orchestra\}

\{Boston Pops Orchestra DVD\}

\{Bayerischer Rundfunk Orchester\}

Names. Access points representing contributors. Searches:

\{filmed operas\}

\{Beethoven Bernstein\}

\{Mozart Da Ponte operas\}

\{Bach Busoni arranged\}

Names. Personal, corporate, and conference names in direct order or where subfields other than $\$$ a are needed for accurate linking or identification. Authorized access points:

100 l\# Adams, John, \$d 1947-

100 1\# Williams, John, $\$ \mathrm{~d} 1932-$

100 0\# Madonna, $\$ \mathrm{~d} 1958$ -

100 0\# Hildegard, \$c Saint, \$d 1098-1179

100 0\# Alfonso \$b X, \$c King of Castile and Leon, \$d 1221-1284

100 1\# Beach, H. H. A., \$c Mrs., \$d 1867-1944

100 1\# Bériot, Ch. de \$q (Charles), \$d 1802-1870

110 2\# Mariinskiǐ teatr (1991- ). \$b Balet

110 2\# Metropolitan Opera (New York, N.Y.). \$b Orchestra

111 2\# Bayreuther Festspiele. \$e Orchester

111 2\# Aspen Music Festival. \$e Contemporary Ensemble

Titles. Distinctive titles, shown with the composer's name and partial or full authorized form of a work title, paired with a corresponding search for the commonly used English title. Searches:

\{Stravinsky zhar-ptitsa\}

\{Stravinsky firebird $\}$

\{Bach brandenburgische konzerte\}

\{Bach brandenburg concerto\}

\{Vivaldi cimento dell'armonia\}

\{Vivaldi four seasons\}

\{Smetana prodana nevesta\}

\{Smetana bartered bride\} 
Titles. Generic titles, shown with the composer's name and work's nickname, paired with a corresponding search for known elements of the title in the work's authorized access point. Searches:

\{Beethoven moonlight\}

\{Beethoven sonatas piano 14272 \}

\{Tchaikovsky pathetique\}

\{Tchaikovsky symphonies 674$\}$

\{Bach little fugue\}

\{Bach BWV 578\}

Titles. Titles containing additions in subfields following $\$ \mathrm{a} / \$ \mathrm{t}$ (shown with titles in $\$ \mathrm{t}$ ). Authorized access points:

Beethoven, Ludwig van, \$d 1770-1827. \$t Symphonies, \$n no. 3, op. 55, \$r E b major

Beethoven, Ludwig van, \$d 1770-1827. \$t Symphonies, \$n no. 3, op. 55, \$r E b major; \$o arranged

Beethoven, Ludwig van, \$d 1770-1827. \$t Sonatas, \$m piano, \$n no. 14, op. 27, no. 2, \$r C\# minor

Janáček, Leoš, \$d 1854-1928. \$t Věc Makropulos. \$1 English

Mozart, Wolfgang Amadeus, \$d 1756-1791. \$t Zauberflöte. \$s Vocal score. \$l English

Note: "Selections" in $\$ \mathrm{k}$ followed by a full stop typically follows the last subfield in authorized access points for work or compilations of, as in:

Beethoven, Ludwig van, $\$ \mathrm{~d}$ 1770-1827. \$t Symphonies, $\$$ n no. 3, op. 55, $\$ \mathrm{r}$ E b major. $\$ \mathrm{k}$ Selections

Beethoven, Ludwig van, \$d 1770-1827. \$t Symphonies, \$n no. 3, op. 55, \$r E b major. \$k Selections; \$o arranged

Beethoven, Ludwig van, $\$ \mathrm{~d} 1770-1827$. $\$$ t Sonatas, $\$ \mathrm{~m}$ piano. $\$ \mathrm{k}$ Selections

Titles. Titles of parts of works (whole-part), shown with titles in $\$$ t. Authorized access points:

Named parts:

Wagner, Richard, \$d 1813-1883. \$t Ring des Nibelungen. \$p Götterdämmerung

Mozart, Wolfgang Amadeus, \$d 1756-1791. \$t Zauberflöte. \$p Hölle Rache

Schumann, Robert, \$d 1810-1856. \$t Frauenliebe und Leben. \$p Du Ring an meinem Finger

Rossini, Gioacchino, \$d 1792-1868. \$t Guillaume Tell. \$p Ouverture

Marais, Marin, $\$ \mathrm{~d} 1656-1728$. $\$$ t Pièces en trio. $\$ \mathrm{p}$ Suite, $\$ \mathrm{r}$ G minor

Numbered parts:

Bach, Johann Sebastian, \$d 1685-1750. \$t Englische Suiten. \$n Nr. 3

Vivaldi, Antonio, \$d 1678-1741. \$t Cimento dell'armonia e dell'inventione. \$n N. 4

Named parts of numbered parts:

Bach, Johann Sebastian, \$d 1685-1750. \$t Brandenburgische Konzerte. \$n Nr. 3. \$p Allegro

Vivaldi, Antonio, \$d 1678-1741. \$t Cimento dell'armonia e dell'inventione. \$n N. 4. \$p Allegro non molto

Titles. Titles containing repeated subfields in varying configurations; subfield order indicated before each, shown with titles in $\$$ t. Authorized access points:

$\$ t \$ m \$ n \$ n$

Corelli, Arcangelo, \$d 1653-1713. \$t Trio sonatas, \$m violins, continuo, \$n op. 1. \$n No. 4

$\$ t \$ m \$ n \$ n \$ p$

Mendelssohn-Bartholdy, Felix, \$d 1809-1847. \$t Lieder ohne Worte, \$m piano, \$n op. 30. \$n Nr. 6, \$p Venezianisches

Gondellied

$\$ t \$ m \$ n \$ p \$ n \$ p$

Bach, Johann Sebastian, \$d 1685-1750. \$t Sonaten und Partiten, \$m violin, \$n BWV 1001-1006. \$p Partita, \$n no. 2. \$p Chaconne

$\$ t \$ n \$ n$

Bach, Johann Sebastian, \$d 1685-1750. \$t Wohltemperierte Klavier, \$n 1. T. \$n Nr. 1

$\$ t \$ n \$ n \$ p$

Wagner, Richard, \$d 1813-1883. \$t Tristan und Isolde. \$n 3. Aufzug. \$n 1. Szene \$p Mässig langsam

$\$ t \$ n \$ p \$ n$

Brahms, Johannes, \$d 1833-1897. \$t Lieder und Gesänge, \$n op. 63. \$p Junge Lieder, \$n no. 1

$\$ t \$ n \$ p \$ p$

Palestrina, Giovanni Pierluigi da, \$d 1525?-1594. \$t Masses, \$n book 2. \$p Missa Papae Marcelli. \$p Credo 
$\$ t \$ n \$ r \$ p \$ p$

Beethoven, Ludwig van, \$d 1770-1827. \$t Symphonies, \$n no. 9, op. 125, \$r D minor. \$p Presto. \$p Allegro assai $\$ t \$ p \$ n \$ p$

Bach, Carl Philipp Emanuel, \$d 1714-1788. \$t Versuch über die wahre Art das Clavier zu spielen. \$p Sonaten. \$n Nr. 6. $\$ \mathrm{p}$ Fantasia

$\$ t \$ p \$ p$

Wagner, Richard, \$d 1813-1883. \$t Ring des Nibelungen. \$p Götterdämmerung. \$p Siegfrieds Rheinfahrt $\$ t \$ p \$ p \$ p$

Wagner, Richard, \$d 1813-1883. \$t Ring des Nibelungen. \$p Götterdämmerung. \$p Brünnhildes Schlussgesang. \$p Grane, mein Ross!

Titles. Preferred titles constructed without association names. Authorized access points:

Singin' in the rain (Motion picture)

Beggar's opera

Ah! vous dirai-je, maman

Kleine Präludien und Fugen

Concerto, $\$ \mathrm{~m}$ bassoon, string orchestra, $\$ \mathrm{n}$ GraunWV Cv:XIII:125, \$r F major

Analytic entries. Records likely to contain name-title analytic entries/access points. Searches:

\{Donizetti Mozart\}

\{Mussorgsky Dukas\}

\{Mozart jupiter linz\}

\{cavalleria pagliacci\}

\{soprano arias songs\}

\{music women composers\}

Titles/analytic entries (name-title)/contributor access points. WorldCat examples:

[score] Later symphonies : full orchestral score of Symphonies 35-41. \#1380022

[score] Mannheim symphonists. \#5903406

[CD] Carreras, Domingo, Pavarotti in concert. \#22433437

[CD] The 3 tenors in concert 1994. \#31042466

[CD] Voice of the violin. \#71222188

[DVD] Stravinsky et les Ballet russes. \#451510365

Relationships. Relationship information indicated in relator designators in 100/700 \$e and in relationship information in $700 \$$ i. WorldCat examples:

[score] Rinaldo and Armida / John Eccles. \#769766464

100 1\# Eccles, John, \$d -1735, \$e composer.

700 1\# Dennis, John, \$d 1657-1734, \$e librettist.

700 1\# Plank, Steven Eric, \$e editor.

700 1\# \$i Libretto based on (work): \$a Tasso, Torquato, \$d 1544-1595. \$t Gerusalemme liberata.

[CD] Symphony no. 2 ; Four Shakespeare preludes / Joachim Raff. \#826876216

700 1\# \$3 2nd work \$i Based on (work): \$a Shakespeare, William, \$d 1564-1616. \$t Tempest.

70012 \$i Contains (work): \$a Raff, Joachim, \$d 1822-1882. \$t Orchester-Vorspiel zu Shakespeare’s Sturm.

Subjects. Records likely to contain name and/or name-title headings used as subjects. Searches:

\{Wagner ring nibelungen\}

\{Bach johannespassion\}

Physical description. Records containing multiple subfields $\$ \mathrm{a}$ and $\$ \mathrm{c}$ in a 300 field or multiple occurrences of the 300 field.

WorldCat index command searches:

\{p3:study parts mt:sco li:DLC\} (RDA)

\{p3:miniature parts mt:sco li:DLC\} (AACR2)

\{p3:miniature parts mt:sco yr:201? li:DLC\} (AACR2) 
Physical description. Records containing multiple subfields $\$ \mathrm{a}$ and $\$ \mathrm{c}$ in a 300 field. WorldCat examples:

[score] Theme and variations for flute and string quartet, op. 80 / Amy Beach. \#35170538

3001 miniature score $(37,4$ p.) ; $\$ \mathrm{c} 22 \mathrm{~cm} .+\$ \mathrm{a} 5$ parts ; $\$ \mathrm{c} 28 \mathrm{~cm}$.

[score] Tríptico : for guitar and string quartet / Roberto Sierra. \#24308364

3001 miniature score $(29$ [i.e. 15] p.) : $\$$ b port. ; $\$ \mathrm{c} 23 \times 31 \mathrm{~cm} .+\$ \mathrm{a} 5$ parts ; $\$ \mathrm{c} 31 \mathrm{~cm}$.

[score] A hundred thousand stars / Jake Heggie. \#730034330

3001 score (9 pages) ; \$c $31 \mathrm{~cm} .+\$ \mathrm{a} 1$ vocal score (6 pages) ; $\$ \mathrm{c} 28 \mathrm{~cm}+\$ \mathrm{a} 5$ parts ; $\$ \mathrm{c} 31 \mathrm{~cm}$.

Physical description. Records representing a multiple-format resource likely to contain $\$$ e in a 300 field or in a repeated

300 field; presence of $006,007(\mathrm{~s})$.

\{scores recorded accompaniments\}

\{lead sheets recorded accompaniments\}

\{Jamey Aebersold jazz series\}

Physical description. Records representing a multiple-format resource, likely to contain $\$$ e in a 300 field or a repeated 300 field; presence of 006, 007(s). WorldCat index command searches:

\{mt:sco mt:rec li:DLC\}

$\{$ dx:rda mt:sco mt:cda li:DLC $\}$

\{dx:rda mt:sco mt:dvv li:DLC $\}$

Physical description. Records representing a multiple-format resources represented in $\$$ e in a 300 field or in a repeated 300 field; presence of 006, 007(s). WorldCat examples:

[score accompanied by CD]

Standard vocal literature : soprano . . . / edited by Richard Walters. \#57732332

006 jsgnn $\quad n$

007 s $\$$ b d $\$$ d f $\$$ e u $\$$ f $\$ g$ g $\$$ h n $\$$ i n $\$$ j m $\$$ k m $\$$ n n $\$$ m e $\$ \mathrm{n} \mathrm{u}$

3001 score (136 p.) ; \$c $31 \mathrm{~cm} .+\$ \mathrm{e} 2$ sound discs (digital ; $43 / 4 \mathrm{in}$.)

[score accompanied by CDs]

Complete guide to learning the Irish tin whistle / by Clare McKenna. \#809031465

006 jfmnn $\quad n$

007 s $\$$ b d $\$$ d f $\$$ e s $\$$ f n $\$ g$ g $\$$ h n $\$$ i n $\$$ j m $\$$ k m $\$$ l n $\$$ m e $\$$ n u

3001 score (96 pages) ; \$c $30 \mathrm{~cm}$

3002 audio discs $(53: 25 ; 72: 39)$ : $\$$ b digital, CD audio, stereo.; $\$ \mathrm{c} 43 / 4$ in.

[CD accompanied by DVD]

One by one / Foo Fighters. \#50852655

006 g--- vl

007 s $\$$ b d $\$$ d f $\$$ e s $\$$ f n $\$$ g g $\$$ h n $\$$ i n $\$$ j m $\$$ k m $\$$ n n $\$$ m e $\$$ n d

007 v $\$$ b d $\$$ d c $\$$ e g $\$$ f a $\$$ g i $\$$ h z $\$$ i u

3001 sound disc (55 min.) : \$b digital ; \$c $43 / 4$ in. + \$e 1 videodisc (DVD : sd., col. ; 4 3/4 in.)

[book accompanied by CD and DVD]

Give my poor heart ease : voices of the Mississippi blues / [interviews by] William Ferris. \#317929511

006 jblnn $\quad \mathrm{n}$

$006 \mathrm{~g}--\quad$ vl

007 s $\$$ b d $\$$ d f $\$$ e u $\$$ f n $\$$ g g $\$$ h n $\$$ i n $\$$ j m $\$$ k m $\$$ l n $\$ \mathrm{~m}$ e $\$$ n u

007 v $\$$ b d $\$$ d b $\$$ e v $\$$ f a $\$$ g i $\$$ h z $\$$ i u

300 xiv, 302 p. : \$b ill., map ; \$c 25 cm. + \$e 1 sound disc (4 3/4 in.) + 1 videodisc (DVD : sd., col. ; 4 3/4 in.)

[book accompanied by CD]

The southern journey of Alan Lomax : words, photographs, and music / with an essay by Tom Piazza ; introduction by

William R. Ferris. \#783162479

006 jmunn $\quad n$

007 s $\$$ b d $\$$ d f $\$$ e n $\$$ f n $\$$ g g $\$$ h n $\$$ i n

300134 pages : \$b illustrations ; \$c $25 \mathrm{~cm}+\$ \mathrm{e} 1$ audio disc (digital ; 4 3/4 in.) 
Format. Recordings that contain 34x fields. WorldCat index command searches:

\{dx:rda en:mp3 mt:msr li:DLC\}

\{dx:rda en:analog mt:msr li:DLC\}

Language. Records likely to contain codes in 041 and note indicating the language(s) of translations or accompanying material. Searches:

\{Lieder English French\}

\{songs texts translations\}

\{biographical notes container\}

\{critical report English German score\}

\{symphonies scores English German\}

\{recent researches music scores\}

Language. Records likely to contain coding for single works composed using multiple languages. Searches:

\{Benjamin Britten war requiem\}

\{Carl Orff carmina burana\}

Language. Various language code configurations in the 041 field. WorldCat examples:

[score] Sixth and seventh symphonies / Ludwig van Beethoven. \#2717378

008/35-37 zxx

$0410 \# \$ g$ eng

500 Introductions by W. Altmann and prefaces by Max Unger: p. [iii]-[xiii].

[score] Luisa Miller / Giuseppe Verdi. \#56357984

008/35-37 ita

041 1\# ita $\$$ a eng $\$$ h ita $\$ g$ ita $\$ g$ eng

546 Italian and English words.

500 Includes pref., introd., and critical commentary in Italian and English.

[score] Standard vocal literature : tenor / edited by Richard Walters. \#57733832

008/35-37 eng

041 1\# eng $\$$ a fre $\$$ a ger $\$ a$ ita $\$ a$ spa $\$$ e eng $\$$ e fre $\$$ e ger $\$$ e ita $\$$ e spa $\$ n$ fre $\$ n$ ger $\$ n$ ita $\$ n$ spa $\$$ g eng

546 English, French, German, Italian, and Spanish words; also printed as texts with English translations preceding each song.

[CD] Carreras, Domingo, Pavarotti in concert. \#22433437

008/35-37 ita

041 1\# $\$$ d ita $\$$ d fre $\$ d$ ger $\$$ d spa $\$$ d eng $\$$ d rus $\$$ e ita $\$$ e fre $\$$ e ger $\$$ e spa $\$$ e eng $\$ \mathrm{n}$ ita $\$ \mathrm{n}$ fre $\$ \mathrm{n}$ ger $\$ \mathrm{n}$ spa $\$ \mathrm{~g}$ eng $\$ \mathrm{~g}$ ita

546 Sung in Italian, French, German, Spanish, English, and Russian.

500 Program notes by Monica Rosolen in Italian and English, and texts (except medley) with English translations (29 p. : ports.) inserted in container.

[DVD] Carmen / Georges Bizet . . Metropolitan Opera. \#665177103

041 1\# fre $\$$ a eng $\$ \mathrm{j}$ fre $\$ \mathrm{j}$ ger $\$ \mathrm{j}$ eng $\$ \mathrm{j}$ spa $\$ \mathrm{j}$ chi $\$ \mathrm{~g}$ eng

546 Sung in French; French, German, English, Spanish or Chinese subtitles; spoken commentary in English.

500 Program notes and synopsis in English (20 p. : ports. ; $18 \mathrm{~cm}$.) inserted in container.

Note: notation/alphabet. Records containing $\$$ b in a 546 field. WorldCat examples:

[score] Rinaldo and Armida / John Eccles ; edited by Steven Plank. \#769766464

546 English words.

546 \$b Staff notation.

[score] Messiah / George Frideric Handel ; edited by Alfred Mann. \#19974051

546 English words.

$546 \$$ b Staff notation. 
Note: performer/cast. Determining indexing of the 511 field in an author index. Author index keyword searches:

\{“Tokyo String Quartet"\} (transcribed in 511)

\{“Tokyo Gengaku Shijusodan"\} (form in heading, macrons omitted)

\{“Meat Loaf Aday"\} (transcribed in 511, Fight Club)

\{“Meat Loaf vocalist"\} (form in heading)

\{Stoltzman clarinet $\}$

Note: place/date. Records with recording place/date information recorded in a formatted 518 field. WorldCat examples:

[CD] Maria di Rohan / Donizetti. \#764337591

518 \$o Recorded \$d 2009 October-November \$p Henry Wood Hall, London.

[CD] Symphony no. 6 in E minor / Vaughan Williams. The sea / Bridge. \#832457650

518 \$3 First work \$o Recorded live \$p BBC Proms \$d 16 August 1972.

518 \$3 Second work \$o Recorded live \$p BBC Hoddinott Hall \$p Cardiff Bay, Wales \$d 3 January 2013.

Note: contents. Records containing enhanced contents note in the 505 field. WorldCat examples:

[CD] Ken Burns jazz. \#45588616

$50510 \$ g$ Disc 1: \$t Star dust \$r (Louis Armstrong \& his Orchestra) . . .

The record contains enhanced contents notes (505, with subfields $\$ \mathrm{~g}$, $\$ \mathrm{t}$, and $\$ \mathrm{r}$ present) with titles that do not appear elsewhere in the bibliographic record. Some performers in subfields $\$ \mathrm{r}$ are included in added entries.

[CD] Born this way / Lady Gaga. \#706505238

$50500 \$$ Marry the night -- \$g Born this way -- \$t Government hooker . . .

This record contatins an enhanced contents note with subfields $\$$ t. All titles except "Born this way" do not appear elsewhere in the bibliographic record.

[CD] The 2006 (\#61343821) and 2010 (\#374374691) editions of the Norton Recorded Anthology of Western Music contain enhanced contents notes with titles and composers that do not appear anywhere else in the records.

Edition. Records containing the 254 field (AACR2). WorldCat examples:

[score] Streichquartett, 1997 / Georg Friedrich Haas. \#783120793

254 Studienpartitur.

3001 score (37 p.) ; \$c $30 \mathrm{~cm}$.

[score] A colorful symphony / Robert Xavier Rodriguez. \#37304903

254 Full score.

3001 miniature score (65 p.) ; \$c $31 \mathrm{~cm}$.

Publisher number/publisher. Records recording multiple publishers in repeated 028 fields. Determining display and indexing. WorldCat examples:

[score] Dix mélodies (1878) / Camille Saint-Saëns. \#190861230

028321269 \$b Recital Publications

02820 A C 3904 \$b A.C. Choudens

260 Huntsville, TX : \$b Recital Publications, \$c 2007.

500 Reprint. Originally published: Paris : A.C. Choudens, 1878. Pl. no. A C 3904.

[CD] Paul Chambers. \#54313915

02800 MS-005 \$b Mosaic Records

02800 LP 7 \$b Jazz West

02800 BLP 1564 \$b Blue Note

260 Stamford, CT : \$b Mosaic Records, \$c p2003.

500 Previously released recordings, principally on Jazz West LP 7 (Chambers' music), Blue Note BLP 1534 (Whims of Chambers), Blue Note BLP 1564 (Paul Chambers Quintet), and Blue Note BST 81569 (Bass on top).

[CD] Ken Burns jazz. \#45588616

02800 C5K 61432 \$b Columbia/Legacy

02800 CK 61433--CK 61437 \$b Columbia/Legacy

02800 61432-2 \$b Columbia/Legacy

500 Columbia/Legacy: C5K 61432 (CK 61433--CK 61437). 
Musical sharps, flats. Determining display and search functionality. Searches:

\{Beethoven Ludwig symphonies $355 \mathrm{E}$ flat major\}

\{Beethoven Ludwig symphonies 355 "E flat major"\}

\{Beethoven Ludwig symphonies $355 \mathrm{E} b$ major\}

\{Beethoven Ludwig symphonies 355 "E $b$ major"\}

\{Beethoven Ludwig sonatas piano $1427 \mathrm{CH}$ minor\} (pound sign)

\{Beethoven Ludwig sonatas piano $1427 \mathrm{C} \#$ minor\} (musical sharp)

Diacritics, special characters, sharps, flats. Names or titles containing sharps, flats, diacritics, or characters, shown with titles in \$t. Authorized access points:

Beethoven, Ludwig van, $\$ \mathrm{~d} 1770-1827$. \$t Sonatas, $\$ \mathrm{~m}$ piano, $\$$ n no. 14, op. 27, no. 2, \$r C\# minor

Dvořák, Antonín, \$d 1841-1904. \$t Humoresques, \$m piano, \$n B. 138, \$r F \# major

Dvořák, Antonín, \$d 1841-1904. \$t Symphonies, \$n no. 2, op. 4, \$r B b major

Mozart, Wolfgang Amadeus, \$d 1756-1791. \$t Zauberflöte

Stravinsky, Igor, \$d 1882-1971. \$t Zhar-ptitsa [ligature over the "ts" in "ptitsa"]

Takemitsu, Tōru. \$t Distance de fée

Cage, John. \$t Four ${ }^{3}$

Ligeti, György, \$d 1923-2006. \$t Traümen Sie in Farbe?

Haanstra, Mark, \$d 1974- $\$ \mathrm{t}$ Y $=6$ afr8 of 7?

1+1 (Harmonia Mundi France (Firm))

Phonogram symbol. Records containing the phonogram symbol @ in field $260 \$ \mathrm{c}$ or $264 \# 4 \$ \mathrm{c}$. WorldCat examples:

[CD] Piano trio no. 1 in C minor, 'Poème', op. 8 . . . Shostakovich. \# 729876690

260 London, England: \$b Hyperion Records Ltd., \$c [2011], (D2011.

[CD] Anthems / Anthrax. \#827267522

264 \#1 [New York City] : \$b Megaforce Records, \$c [2013]

264 \#4 \$c (2013

Stopwords/Boolean operators. Determining search functionality. Searches:

\{Bach inventions a minor\}

\{Bach inventions "a minor"

\{Beethoven eroica no 3\}

\{Beethoven eroica "no" 3\} or \{Beethoven eroica "no 3"\} 